\title{
Automated Detection of Near Surface Martian Ice Layers In Orbital Radar Data
}

\author{
Greg J. Freeman \\ The University of Texas at Austin \\ Electrical and Computer Engineering \\ Austin, TX \\ greg.freeman@mail.utexas.edu
}

\author{
Alan C. Bovik \\ The University of Texas at Austin \\ Electrical and Computer Engineering \\ Austin, TX \\ bovik@ece.utexas.edu
}

\author{
John W. Holt \\ The University of Texas at Austin \\ Institute for Geophysics \\ Austin, TX \\ jack@ig.utexas.edu
}

\begin{abstract}
An algorithm is presented to automatically detect near surface ice layers in images from the Shallow Subsurface Radar (SHARAD) on NASA's Mars Reconnaissance Orbiter. Mars' ice-rich Northern Polar Layered Deposits (NPLD) represents an extensive geologic record of climate history. Identifying ice layers in cross-sectional images leads to understanding the three-dimensional structure of ice layers. Scientists have manually identified layers in large data volumes, but the automated algorithm will allow studying more images from over a thousand orbital crossings. A unique coordinate transformation, based upon the surface reflection, makes subsequent filtering and detection more effective on near surface layers. Results show promising capabilities for automatically detecting ice layers on Mars.
\end{abstract}

Keywords-Synthetic Aperture Radar Sounding, Image Processing, Mars Northern Polar Layered Deposits

\section{INTRODUCTION}

The Shallow Subsurface Radar (SHARAD) on NASA's Mars Reconnaissance Orbiter [1] has been acquiring data at Mars since late 2006. The coherent radar sounder collects data that shows cross sections of the Mars subsurface where penetration is sufficient and dielectric contrasts exist. The subsurface layering in the ice-rich Mars Northern Polar Layered Deposits (NPLD) represent the most extensive geologic record of climate history on Mars and is an excellent target for radar sounding studies [2]. The SHARAD instrument has acquired data from over a thousand orbital crossings of the NPLD. Knowing the three-dimensional structure of the interior gives geologists important constraints on how the polar ice was deposited, and how it may have been deformed and eroded over time. Scientists studying these images have manually interpreted and labeled many layers in large data volumes, but the manually intensive and repetitive nature of identifying specific layers calls for an automated signal processing method of detecting and labeling layers. In this paper we present an algorithm that detects and segments shallow layers in radar images.

\section{SHARAD}

The SHARAD instrument is a synthetic aperture radar (SAR) sounder orbiting Mars. The radar uses linear frequency modulation (LFM) in the range of $15 \mathrm{MHz}$ to $25 \mathrm{MHz}$. The radar antenna produces a wide beam width transmitting and receiving energy toward the Martian surface. Along the direction that the satellite travels, or the along-track direction, the SAR processing algorithm reconstructs the reflections spatially. In the cross-track direction (perpendicular to the along-track direction) signals are not focused, but nadir reflections are much stronger than off-nadir reflections over level terrain, so images show a good representation of the mars subsurface. Fig. 1 shows a diagram of the imaging geometry and Fig. 2 shows an example image from SHARAD. For this experiment, magnitude imagery scaled on a decibel scale was available. In the downrange direction, samples represent $37.5 \mathrm{~ns}$, but due to the bandwidth only $100 \mathrm{~ns}$ features can be resolved. This translates to resolving $15 \mathrm{~m}$ in free-space or $8.4 \mathrm{~m}$ in ice, assuming a dielectric constant of 3.15 . The data in this experiment had an along-track resolution of $300 \mathrm{~m}$ after onedimensional focusing. This aspect ratio yielded image pixels that are much wider than tall. Understanding the aspect ratio was important in subsequent image processing.

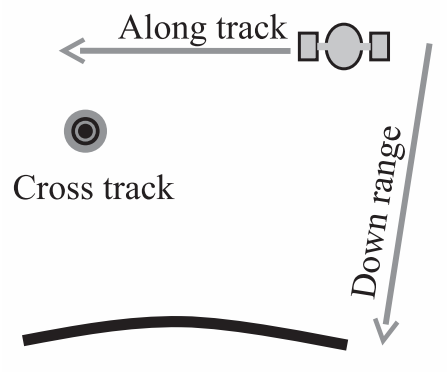

Figure 1. Imaging geometry.

\section{ICE LAYER DETECTION}

\section{A. Motivation}

Layers in the approximately 2-km-thick NPLD range in thickness from tens of centimeters to several meters as observed in optical data, where the distinction is assumed to derive from changes in dust content related to climate. The resulting vertical changes in dielectric properties are thought to be responsible for radar reflections [3]. While SHARAD cannot resolve all optically observed layers, it 


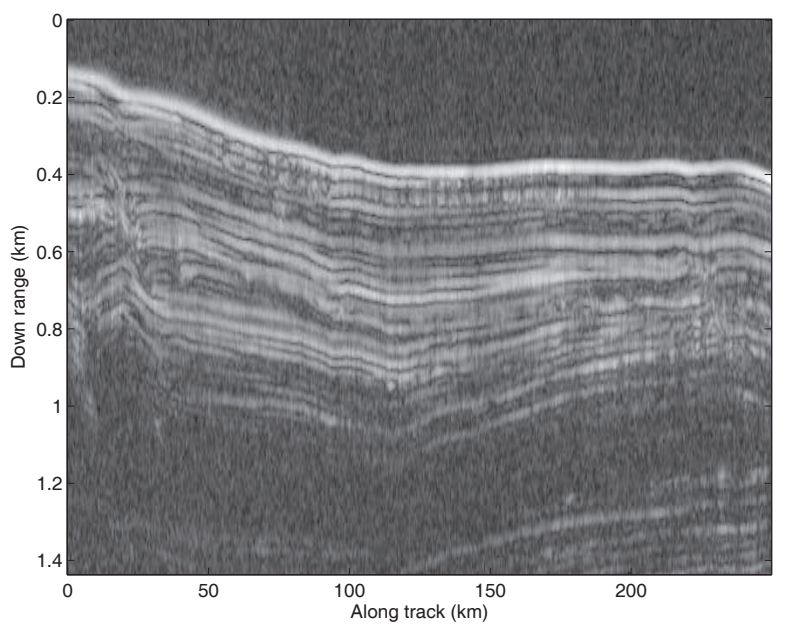

Figure 2. Example image: $A_{\text {image }}\left(x, y^{\prime}\right)$

appears that radar reflections follow the same geometry as packets of optical layers [4]; therefore, the mapping of radar layers serves as a proxy for optical layers with the distinct advantage of imaging throughout the deposits, rather than only at limited exposures by optical instruments. Exposures are locations on the planet surface where layering is visible.

Mapping individual layers across the many intersecting SHARAD orbits that cross the polar regions provides the ability to construct gridded surfaces representing paleoNPLD surfaces, or at least close approximations. This is important for studying the evolution of the polar deposits, and previously has been accomplished manually using a proprietary, interactive software designed for seismic data analysis. Users interactively pick points along a radar image layer (e.g., Fig. 2 ) and the software assists users to an extent by finding nearby peaks and connecting user-selected control points. The manual software can also display intersecting orbit passes and their associated layer pick positions to facilitate correlating layers across multiple orbital passes.

With thousands of orbits and tens of layers in each orbit, the process of manually mapping individual layers becomes a major limitation in the analysis of SHARAD data for such studies. Also, it is often not possible to correlate layers across large regions due to interruptions in layer geometry caused by non-nadir surface reflections of sloping terrain. An algorithm to detect and identify specific layers would greatly assist in this process and provide the basis for automated layer tracking across intersecting orbits. If combined with the complementary problem of automatically correlating layers in intersecting orbital passes, it may be feasible to match and identify layers over large areas, even if they are not contiguous. This would greatly enhance the interpretation of radar data for geologic studies.

\section{B. Implementation}

In observing the near surface layers, layers would appear faint at times, but a human observer can detect the presence of a layer because the layers are parallel to one another. Also near the surface, layers are parallel to the surface. The surface reflection is very strong and layer intensity generally decreases as depth increases, but some deep layers contain a greater concentration of reflective materials and reflect brighter than shallow layers that are less reflective. There is also a large amount of what appears to be Gaussian noise. Using observations about how humans detect ice layers aids the development of automated algorithms that mimic these behaviors.

By using the surface reflection as a reference, the image space was transformed into surface depth. This transformation changed wavy layers near the surface into flat horizontal layers. With horizontal layers, filtering was possible to continue areas where the layering was faint. Next, thresholding and morphological processing was applied to discard unclear layers. Finally, the layers were transformed back into image space.

1) Depth Transformation: Since a strong surface reflection is easy to detect we can define a function $f(x)$, which is the surface height. For each image column, we can shift the columns up so that the surface reflection is at the top of the image. If the radar image is defined as $A_{\text {image }}\left(x, y^{\prime}\right)$, then we can compute the transform as (1). The $x$ dimension is along-track and $y^{\prime}$ is downrange. Because of the extreme pixel aspect ratio $(5.6 \mathrm{~m} \times 300 \mathrm{~m})$, neighboring pixels on the left and right can be ignored and each column can be processed independently.

$$
A_{\tau}(x, y)=A_{\text {image }}\left(x, \frac{y^{\prime}-f(x)}{\sqrt{\varepsilon_{r}}}\right), \varepsilon_{r}=3.15
$$

In the transformed image, the $y$ axis becomes relative to the Mars surface instead of relative to the Mars ellipsoid datum. The $y^{\prime}$ axis of Fig. 2 shows distance to simplify interpretation, but the distance is assuming free-space. Radar energy propagates slower underground. Since $A_{\tau}$ is uniformly underground, we scaled the $y$ axis to distance in ice. The notation $y$ verses $y^{\prime}$ is used to signify the different axis scale.

2) Filtering: Since the downrange sampling rate is finer than the radar resolution size and there is considerable noise in the image, a low-pass Gaussian blur was applied to the image. Also to normalize areas with high reflectivity with areas of low reflectivity, a high-pass filter was applied. This revealed that mid-band spatial frequencies provided the most important information content to detect ice layers. Equations (2)-(4) show the filtering.

$$
H_{\sigma}(x, y)=\frac{1}{2 \pi \sigma^{2}} \exp \left(-\left(\frac{x^{2}+y^{2}}{2 \sigma^{2}}\right)\right)
$$




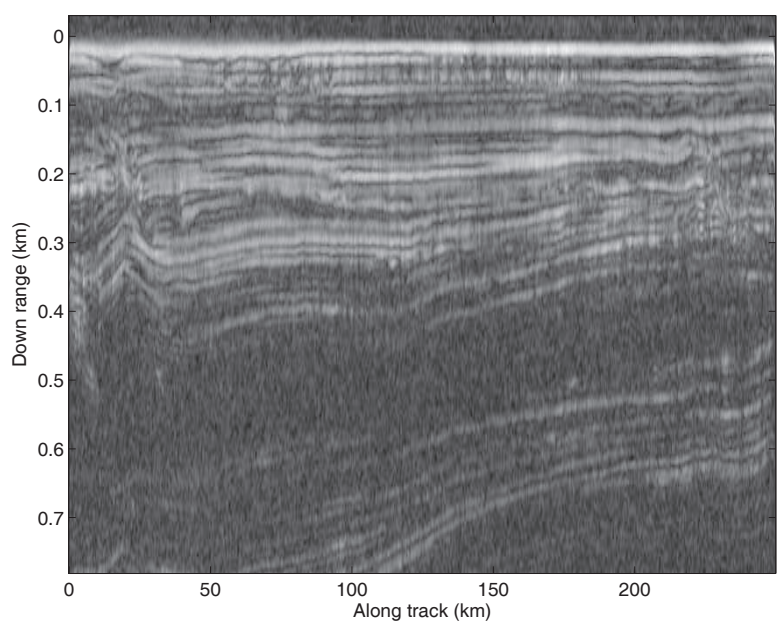

Figure 3. Image transformed to depth from surface: $A_{\tau}(x, y)$

$$
\begin{gathered}
H_{\text {low-pass }}(x, y)=\left.H_{\sigma}(x, y)\right|_{\sigma=1 p x} \\
H_{\text {high-pass }}(x, y)=1-\left.H_{\sigma}(x, y)\right|_{\sigma=3 p x}
\end{gathered}
$$

The spatial filters use the same pixel size for $x$ and $y$ directions which gives the filter the same oblong stretched shape in real space as the image pixels. This seemed undesirable initially, but when considering the features that we are interested in, it works well. The visible ice layers extend for hundreds of kilometers, but are only 5-15 meters thick. In addition to band-pass filtering (through the combination of a low and high-pass filter), we created a filter matched to the shape of the ice layers. The ice layer filter $H_{\text {ice }}$ looked for reflected horizontal ridges that were 3 pixels (about 9 meters) thick as shown in (5). The filter extends along approximately 6 kilometers. Fig. 4 shows the image after filtering.

$$
\begin{gathered}
H_{\text {ice }}(x, y)=\left[\begin{array}{ccccc}
-1 & -1 & \cdots & -1 & -1 \\
+1 & +1 & \cdots & +1 & +1 \\
+1 & +1 & \cdots & +1 & +1 \\
+1 & +1 & \cdots & +1 & +1 \\
-1 & -1 & \cdots & -1 & -1
\end{array}\right] \\
H=H_{\text {high-pass }} * H_{\text {low-pass }} * H_{\text {ice }} \\
A_{\text {filtered }}(x, y)=A_{\text {transformed }}(x, y) * H
\end{gathered}
$$

3) Detection: The detection step applied a threshold and then morphological processing to remove unclear or unlikely layers. Using connected components analysis, segments with a small area were discarded.

$$
A_{\text {binary }}(x, y)=A_{\text {filtered }}(x, y)>t
$$

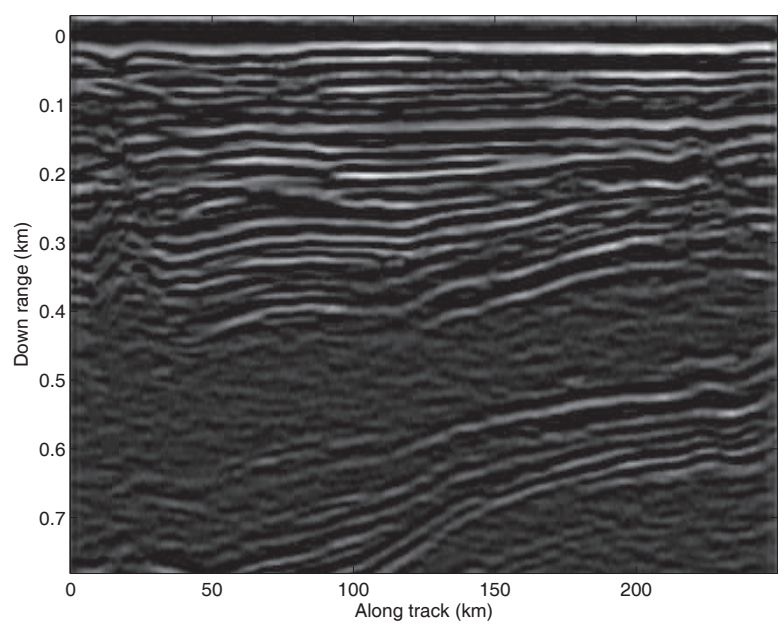

Figure 4. Filtered image: $A_{\text {filtered }}(x, y)$

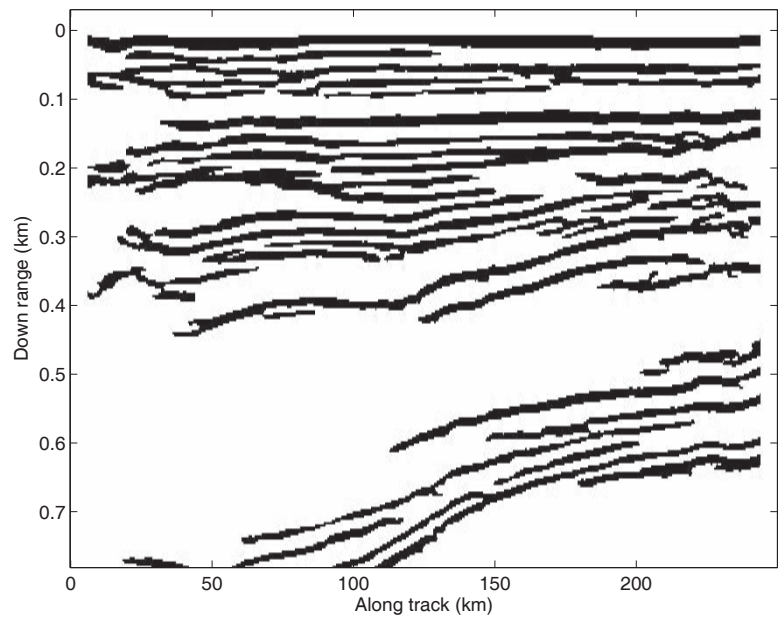

Figure 5. Detected layers: $A_{\text {layers }}(x, y)$

4) Reverse Transformation: The inverse transform returned the detected layers back to image space. In image space, the $y^{\prime \prime}$ axis becomes relative to the Mars ellipsoid datum instead of depth below the surface. We must again account for the dielectric differences between ice and freespace. Fig. 6 shows the resulting layers.

$$
A_{\tau^{-1}}\left(x, y^{\prime \prime}\right)=A_{\text {layers }}(x, y+f(x))
$$

\section{RELATED WORK}

The most similar work was by Fahnestock [5], which used a signal processing algorithm to track Greenland sheets of ice from an airborne ground-penetrating radar (GPR). The Mars problem is more challenging because the Mars SHARAD data has higher noise levels and discontinuities that will throw off a peak following algorithm as used in the Greenland work. There are a number of other related 


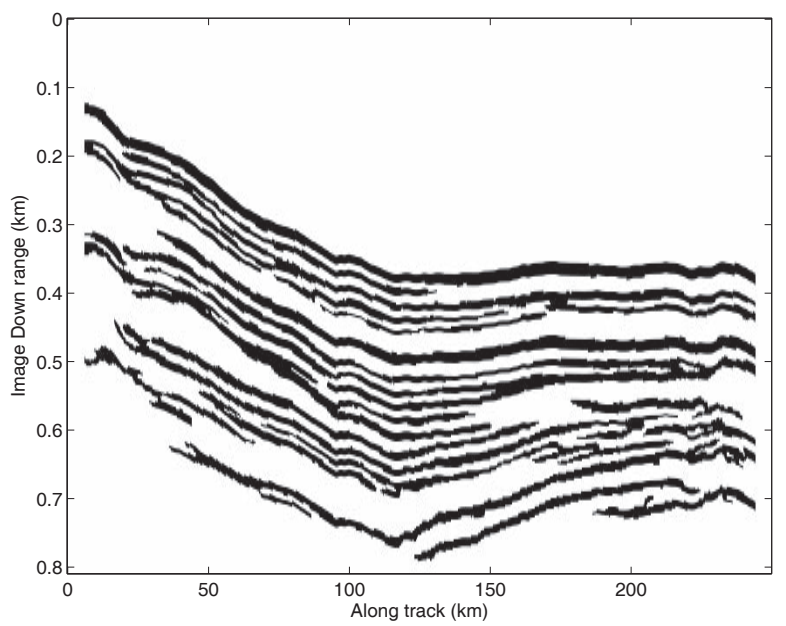

Figure 6. Layers following reverse transformation: $A_{\tau^{-1}}\left(x, y^{\prime \prime}\right)$

works that solve different problems. In [6], [7], Deng and Clausi developed an image processing algorithm that detects floating icebergs from overhead SAR imagery of the ocean surface. While this work detected ice and involved radar images, it used a much different imaging geometry so the detected features were much different. With regards to the image processing problem, similar looking features can be found in medical and fingerprint images. Pattichis applied amplitude modulation (AM) frequency modulation (FM) decomposition to segment similar feature shapes in medical imagery [8]. Fingerprint image analysis has also applied AM-FM models [9], [10]. There is work in seismic and GPR processing to automatically detect underground geological features, but no other work was found that tracks layers over horizontal distances and thus does not assist inferring underground geometry from the wealth of SHARAD data. Of course, there is considerable literature on segmenting various features in optical images and other modalities [11], [12], [13], [14], but it does not directly address the problem either.

\section{CONCLusion}

The initial results show promising capabilities for automatically detecting ice layers on Mars. Future research will lead into applying alternative methods such as active appearance models and AM-FM decomposition. Automatic detection of ice layers in single images of the subsurface leads to the complementary problem of automatically correlating layers in multiple orbital passes to understand the three-dimensional structure of subsurface ice layers. In this paper, we focused on post-processing SAR images, but given dense radar data, improved radar processing may be possible based on variants of interferometric SAR processing.

\section{REFERENCES}

[1] R. Seu et al., "SHARAD sounding radar on the Mars Reconnaissance Orbiter," Journal of Geophysical Research, vol. 112, no. E05S05, 2007.

[2] R. Phillips, et al., "Mars north polar deposits: Stratigraphy, age, and geodynamical response," Science, vol. 320, no. 5880, pp. 1182-1185, 2008.

[3] D. Nunes and R. Phillips, "Radar subsurface mapping of the polar layered deposits on Mars," Journal of Geophysical Research, vol. 111, no. E06S21, 2006.

[4] S. M. Milkovich, J. J. Plaut, A. Safaeinili, G. Picardi, R. Seu, and R. J. Phillips, "Stratigraphy of Promethei Lingula, south polar layered deposits, Mars, in radar and imaging data sets," Journal of Geophysical Research, vol. 114, no. E03002, 2009.

[5] M. Fahnestock, W. Abdalati, S. Luo, and S. Gogineni, "Internal layer tracing and age-depth-accumulation relationships for the northern Greenland ice sheet," Journal of Geophysical Research-Atmospheres, vol. 106, no. D24, 2001.

[6] H. Deng and D. Clausi, "Unsupervised segmentation of synthetic aperture radar sea ice imagery using a novel Markov random field model," IEEE Transactions on Geoscience and Remote Sensing, vol. 43, no. 3, pp. 528-538, 2005.

[7] D. Clausi, "Texture segmentation of SAR sea ice imagery," University of Waterloo, Waterloo, Ontario, Canada N2L 3G1, 1996.

[8] M. Pattichis, C. Pattichis, M. Avraam, A. Bovik, and K. Kyriacou, "AM-FM texture segmentation in electron microscopic muscle imaging," IEEE transactions on medical imaging, vol. 19, no. 12, pp. 1253-1257, 2000.

[9] M. Pattichis and A. Bovik, "Latent Fingerprint Analysis Using an AM-FM Model," Automatic Fingerprint Recognition Systems, pp. 317-338.

[10] S. Acton, D. Mukherjee, J. Havlicek, and A. Bovik, "Oriented texture completion by AM-FM reaction-diffusion," IEEE Transactions on Image Processing, vol. 10, no. 6, pp. 885896, 2001.

[11] J. Shotton, M. Johnson, R. Cipolla, and J. Kawasaki, "Semantic texton forests for image categorization and segmentation," in Proc. CVPR, 2008.

[12] L. Fei-Fei and P. Perona, "A bayesian hierarchical model for learning natural scene categories," in Proc. CVPR, 2005.

[13] M. Maire, P. Arbelaez, C. Fowlkes, and J. Malik, "Using Contours to Detect and Localize Junctions in Natural Images," in Proc. CVPR, 2008.

[14] S. Gould, J. Rodgers, D. Cohen, G. Elidan, and D. Koller, "Multi-class segmentation with relative location prior," International Journal of Computer Vision, vol. 80, no. 3, pp. 300-316, 2008. 